\title{
Scheme for deterministic Bell-state-measurement-free quantum teleportation
}

\author{
Ming Yang, ${ }^{1, *}$ and Zhuo-Liang $\mathrm{Cao}^{1, \text {, }}$ \\ ${ }^{1}$ Anhui Key Laboratory of Information Material \& Devices,School of Physics \& Material Science, \\ Anhui University, Hefei, 230039, PRChina
}

\begin{abstract}
A deterministic teleportation scheme for unknown atomic states is proposed in cavity QED. The Bell state measurement is not needed in the teleportation process, and the success probability can reach 1.0. In addition, the current scheme is insensitive to the cavity decay and thermal field.
\end{abstract}

PACS numbers: 03.67.Hk, 03.67.Mn, 03.67.Pp

Quantum teleportation is one of the most important applications of quantum entanglement. In quantum teleportation process, unknown quantum information can be transmitted from a sender to a receiver without the transmission of the carrier of the quantum information [1]. In the original scheme for quantum teleportation, the sender and the receiver share a maximally entangled state quantum channel. To realize the teleportation, the sender must operate a joint Bell state measurement on the particle that carries the unknown quantum information and one of the entangled particles he possesses. Then the sender will inform the receiver his measurement result. Finally, the receiver will operate a single particle transformation on the particle he possesses to transform the state into the unknown state to be teleported based on the send's measurement result.

Recently, there is rapid progress in quantum teleportation of unknown photon states. D.Bouwmeester et al proposed the first experimental demonstration for the teleportation of unknown polarization photon states 2]. Then the realization of the deterministic teleportation of unknown continuous quantum variables states of a beam of light was reported[3]. Pan et al proposed the experimental realization of freely propagating teleported qubits [4].

We find that teleportation has been demonstrated in the domain of photon. But, for the teleportation of unknown atomic states, it is not the case. In cavity QED, several schemes have been proposed for the teleportation of unknown atomic states. Cirac et al have made a proposal for quantum teleportation of an atomic state by using two additional atomic levels of one of the correlated pair [5]. Bose et al proposed a unique proposal for the teleportation of atomic states via cavity decay $\underline{6}]$. Some other teleportation schemes have been proposed in cavity QED 7, 8, 9, 10, 11]. A more feasible scheme for quantum information processing has been proposed [12], where quantum teleportation of unknown atomic state is realized by the dispersive interaction between two atoms and a single-mode cavity. Because there is no exchange of energy between atoms and cavity mode during the in-

\footnotetext{
*Electronic address: mingyang@ahu.edu.cn
}

${ }^{\dagger}$ Electronic address: zlcao@ahu.edu.cn teraction, the scheme is insensitive to cavity decay. Recently, superb progress has been made in the realization of teleportation of atomic states. Riebe et al and Barrett et al have experimentally implemented the teleportation of the states of $\mathrm{Ca}^{+}\left[13\right.$ and $\mathrm{Be}^{+}[14$ respectively.

From analysis, we find that the key steps in quantum teleportation are generation of quantum channel and realization of the join Bell state measurement. Generation of entangled atomic states has been implemented in experiment 15]. But the joint Bell state measurement for atomic states is still a bottle-neck for the quantum teleportation. Although the joint operation has been realized in Refs 13, 14], the operations needed there are so complex. To overcome the difficulty of Bell state measurement, Zheng proposed a teleportation scheme in cavity QED, where the joint Bell state measurement is not needed [16]. So the scheme is more feasible than the others. But, in the scheme, the interaction between atoms and cavity is a resonant one, and the cavity decay and the thermal field will affect the scheme strongly. So Ye and Guo proposed another teleportation scheme without Bell state measurement 17]. In their scheme, the interaction is a dispersive one 12], so the effect of cavity decay has been eliminated. But the effect of thermal field still exists. The success probabilities of these two schemes are 0.25 and 0.5 respectively, i.e. they are all probabilistic schemes.

In this paper, following a recent contribution on entanglement generation [18], we propose a deterministic teleportation scheme without Bell state measurement, from which the effects of thermal field and cavity decay are all eliminated. The success probability is 1.0 .

Next we will discuss the teleportation process in more details. The sender possesses an atom 1 in unknown state:

$$
|\Phi\rangle_{1}=\alpha|e\rangle_{1}+\beta|g\rangle_{1}
$$

where $|e\rangle$ and $|g\rangle$ are the excited state and ground state of atoms. Before considering the teleportation process per se, we will consider the generation of the quantum channel. Consider two atoms 2, 3, which are all prepared in ground states initially. To generate two-atom maximally entangle states, a single-mode cavity must be introduced. Atoms 2, 3 will be sent through the cavity. At the same time, the two atoms are driven by a classical field. Then the interaction between atoms and the 
single-mode cavity can be described as follow:

$$
\begin{aligned}
H & =\omega_{0} \sum_{j=1}^{2} S_{z, j}+\omega_{a} a^{+} a+\sum_{j=1}^{2}\left[g\left(a^{+} S_{j}^{-}+a S_{j}^{+}\right)\right. \\
& \left.+\Omega\left(S_{j}^{+} e^{-i \omega_{d} t}+S_{j}^{-} e^{i \omega_{d} t}\right)\right]
\end{aligned}
$$

where $\omega_{0}, \omega_{a}$ and $\omega_{d}$ are atomic transition frequency $(e \leftrightarrow g)$, cavity frequency and the frequency of driving field respectively, $a^{+}$and $a$ are creation and annihilation operators for the cavity mode, $g$ is the coupling constant between atoms and cavity mode, $S_{j}^{-}=|g\rangle_{j}\langle e|$, $S_{j}^{+}=|e\rangle_{j}\langle g|, S_{z, j}=\frac{1}{2}\left(|e\rangle_{j}\langle e|-| g\rangle_{j}\langle g|\right)$ are atomic operators, and $\Omega$ is the Rabi frequency of the classical field. We consider the case $\omega_{0}=\omega_{d}$. In the interaction picture, the evolution operator of the system is [16]:

$$
U(t)=e^{-i H_{0} t} e^{-i H_{e f f} t},
$$

where $H_{0}=\sum_{j=1}^{2} \Omega\left(S_{j}^{+}+S_{j}^{-}\right), H_{e f f}$ is the effective Hamiltonian. In the large detuning $\delta \gg \frac{g}{2}$ and strong driving field $2 \Omega \gg \delta, g$ limit, the effective Hamiltonian for this interaction can be described as follow [16]:

$$
\begin{aligned}
H_{e f f} & =\lambda\left[\frac{1}{2} \sum_{j=1}^{2}\left(|e\rangle_{j}\langle e|+| g\rangle_{j}\langle g|\right)\right. \\
& \left.+\sum_{j, k=1, j \neq k}^{2}\left(S_{j}^{+} S_{k}^{+}+S_{j}^{+} S_{k}^{-}+\text {H.c. }\right)\right],
\end{aligned}
$$

where $\lambda=\frac{g^{2}}{2 \delta}$ with $\delta$ being the detuning between atomic transition frequency $\omega_{0}$ and cavity frequency $\omega_{a}$. From the form of the effective Hamiltonian, we conclude that the interaction Hamiltonian is independent of the photon number of the cavity field. In addition, there is no exchange of energy between atoms and cavity mode. So the the effects of cavity decay and thermal field are all eliminated.

After interaction time $t$, the state of the two atoms 2, 3 will undergo the following evolution:

$$
\begin{aligned}
|g\rangle_{2}|g\rangle_{3} \longrightarrow & e^{-i \lambda t}\left[\cos \lambda t\left(\cos \Omega t|g\rangle_{2}-i \sin \Omega t|e\rangle_{2}\right)\right. \\
& \times\left(\cos \Omega t|g\rangle_{3}-i \sin \Omega t|e\rangle_{3}\right) \\
& -i \sin \lambda t\left(\cos \Omega t|e\rangle_{2}-i \sin \Omega t|g\rangle_{2}\right) \\
& \left.\times\left(\cos \Omega t|e\rangle_{3}-i \sin \Omega t|g\rangle_{3}\right)\right]
\end{aligned}
$$

Choosing the interaction time $t$, we can let $\lambda t=\frac{\pi}{4}$. Then the condition $\Omega t=\pi$ can be realized by modulating the driving field appropriately. Then the atoms 2,3 will be left in:

$$
|\Phi\rangle_{23}=\frac{1}{\sqrt{2}}\left(|e\rangle_{2}|e\rangle_{3}+i|g\rangle_{2}|g\rangle_{3}\right),
$$

which will be used as quantum channel. Suppose that the atoms 2, 3 have been distributed among the sender (Alice) and the receiver (Bob). To teleport the state of atom 1 to atom 3, Alice will send the two atoms 1, 2 into a single-mode cavity. At the same time, the two atoms 1, 2 are driven by a classical field. This interaction has been described in Equations (2) 4). The evolution of the total system can be expressed as:

$$
\begin{aligned}
& \left(\alpha|e\rangle_{1}+\beta|g\rangle_{1}\right) \otimes \frac{1}{\sqrt{2}}\left(|e\rangle_{2}|e\rangle_{3}+i|g\rangle_{2}|g\rangle_{3}\right) \\
& \longrightarrow \frac{1}{\sqrt{2}} \alpha e^{-i \lambda t^{\prime}}\left[\cos \lambda t^{\prime}\left(\cos \Omega t^{\prime}|e\rangle_{1}-i \sin \Omega t^{\prime}|g\rangle_{1}\right) \times\left(\cos \Omega t^{\prime}|e\rangle_{2}-i \sin \Omega t^{\prime}|g\rangle_{2}\right)\right. \\
& \left.-i \sin \lambda t^{\prime}\left(\cos \Omega t^{\prime}|g\rangle_{1}-i \sin \Omega t^{\prime}|e\rangle_{1}\right) \times\left(\cos \Omega t^{\prime}|g\rangle_{2}-i \sin \Omega t^{\prime}|e\rangle_{2}\right)\right]|e\rangle_{3} \\
& +\frac{i}{\sqrt{2}} \alpha e^{-i \lambda t^{\prime}}\left[\cos \lambda t^{\prime}\left(\cos \Omega t^{\prime}|e\rangle_{1}-i \sin \Omega t^{\prime}|g\rangle_{1}\right) \times\left(\cos \Omega t^{\prime}|g\rangle_{2}-i \sin \Omega t^{\prime}|e\rangle_{2}\right)\right. \\
& \left.-i \sin \lambda t^{\prime}\left(\cos \Omega t^{\prime}|g\rangle_{1}-i \sin \Omega t^{\prime}|e\rangle_{1}\right) \times\left(\cos \Omega t^{\prime}|e\rangle_{2}-i \sin \Omega t^{\prime}|g\rangle_{2}\right)\right]|g\rangle_{3} \\
& +\frac{1}{\sqrt{2}} \beta e^{-i \lambda t^{\prime}}\left[\cos \lambda t^{\prime}\left(\cos \Omega t^{\prime}|g\rangle_{1}-i \sin \Omega t^{\prime}|e\rangle_{1}\right) \times\left(\cos \Omega t^{\prime}|e\rangle_{2}-i \sin \Omega t^{\prime}|g\rangle_{2}\right)\right. \\
& \left.-i \sin \lambda t^{\prime}\left(\cos \Omega t^{\prime}|e\rangle_{1}-i \sin \Omega t^{\prime}|g\rangle_{1}\right) \times\left(\cos \Omega t^{\prime}|g\rangle_{2}-i \sin \Omega t^{\prime}|e\rangle_{2}\right)\right]|e\rangle_{3} \\
& +\frac{i}{\sqrt{2}} \beta e^{-i \lambda t^{\prime}}\left[\cos \lambda t^{\prime}\left(\cos \Omega t^{\prime}|g\rangle_{1}-i \sin \Omega t^{\prime}|e\rangle_{1}\right) \times\left(\cos \Omega t^{\prime}|g\rangle_{2}-i \sin \Omega t^{\prime}|e\rangle_{2}\right)\right. \\
& \left.-i \sin \lambda t^{\prime}\left(\cos \Omega t^{\prime}|e\rangle_{1}-i \sin \Omega t^{\prime}|g\rangle_{1}\right) \times\left(\cos \Omega t^{\prime}|e\rangle_{2}-i \sin \Omega t^{\prime}|g\rangle_{2}\right)\right]|g\rangle_{3} .
\end{aligned}
$$


TABLE I: The results of the teleportation scheme. M.R. denotes the measurement result on atoms 1, 2, Operation denotes the operation needed for the receiver.

\begin{tabular}{lcr}
\hline \hline M.R. & $|\Psi\rangle_{3}$ & Operation \\
$|e\rangle_{1}|e\rangle_{2}$ & $\frac{1}{2}\left(\alpha|e\rangle_{3}+\beta|g\rangle_{3}\right)$ & $\mathrm{I}$ \\
$|g\rangle_{1}|g\rangle_{2}$ & $\frac{1}{2}\left(\alpha|e\rangle_{3}-\beta|g\rangle_{3}\right)$ & $\sigma_{z}$ \\
$|e\rangle_{1}|g\rangle_{2}$ & $\frac{1}{2}\left(\alpha|g\rangle_{3}-\beta|e\rangle_{3}\right)$ & $\sigma_{y}$ \\
$|g\rangle_{1}|e\rangle_{2}$ & $\frac{1}{2}\left(\alpha|g\rangle_{3}+\beta|e\rangle_{3}\right)$ & $\sigma_{x}$ \\
\hline \hline
\end{tabular}

collapse into the following state:

$$
\begin{aligned}
|\Psi\rangle_{3} & =\frac{1}{\sqrt{2}} \alpha e^{-i \lambda t^{\prime}}\left(e^{-i \lambda t^{\prime}} \cos ^{2} \Omega t^{\prime}+i \sin \lambda t^{\prime}\right)|e\rangle_{3} \\
& -\frac{i}{\sqrt{2}} \beta e^{-i \lambda t^{\prime}}\left(e^{-i \lambda t^{\prime}} \sin ^{2} \Omega t^{\prime}+i \sin \lambda t^{\prime}\right)|g\rangle_{3} \\
& +\frac{1}{2 \sqrt{2}} \alpha e^{-2 i \lambda t^{\prime}} \sin 2 \Omega t^{\prime}|g\rangle_{3} \\
& -\frac{i}{2 \sqrt{2}} \beta e^{-2 i \lambda t^{\prime}} \sin 2 \Omega t^{\prime}|e\rangle_{3} .
\end{aligned}
$$

If the interaction time satisfies the condition $\lambda t^{\prime}=\frac{\pi}{4}$ and $\Omega t^{\prime}=\pi$, the state in Equation (8) becomes:

$$
\left|\Psi^{\prime}\right\rangle_{3}=\frac{1}{2}\left(\alpha|e\rangle_{3}+\beta|g\rangle_{3}\right) .
$$

So the teleportation succeeds with probability $\frac{1}{4}$. If the measurement results are $|g\rangle_{1}|g\rangle_{2},|g\rangle_{1}|e\rangle_{2},|e\rangle_{1}|g\rangle_{2}$, the teleportation also can succeed with probability $\frac{1}{4}$ (As depicted in the Table.1). That is to say the total success probability is 1.0 , which is more higher than that of the previous ones. Table. 1 lists the measurement results on atoms 1, 2, the result state of atom 3 and the operation needed for the receiver to convert the state of atom 3 into the initial state of atom 1 .
Next, Consider the feasibility of the current scheme. Because the scheme is insensitive to cavity decay and thermal field, the scheme only must be completed within the radiative time of the atoms. For Rydberg atoms with principal quantum numbers 50 and 51 , the radiative time is $T_{a}=3 \times 10^{-2} s$. From the analysis in reference [12], the interaction time is on the order $t \simeq 2 \times 10^{-4} s$, which is much shorter than the atomic radiative time $T_{a}$. So our scheme is reliable by using cavity QED techniques.

In conclusion, we proposed a deterministic scheme for the teleportation of unknown atomic states, where the Bell state measurement is not needed. The distinct advantage of the current scheme is that, not only teleportation of unknown atomic states can be realized without the Bell state measurement, but also the success probability can reach 1.0 with fidelity being 1.0. The dispersive interaction between two driven atoms and a single-mode cavity used here makes the current scheme possess another distinct advantage, i.e. the effects of cavity decay and thermal field are all eliminated. But, there is still a disadvantage here. We must distinguish the two atoms 1 , 2 after they flying out of the cavity. Fortunately, Riebe et al 13] have developed a technique to address any specified target ion using tightly focused laser beams and to 'hide' the remaining ions from the target ion's fluorescence by changing their internal states so that they are insensitive to the fluorescent light. So the scheme is feasible in the current technology.

\section{Acknowledgments}

This work is supported by the Natural Science Foundation of the Education Department of Anhui Province under Grant No: 2004kj005zd and Anhui Provincial Natural Science Foundation under Grant No: 03042401 and the Talent Foundation of Anhui University.
[1] C. H. Bennett, G. Brassard, C. Crépeau, R. Jozsa, A. Peres, \& W. K.Wootter, Phys. Rev. Lett. 70, 1895 (1993).

[2] D. Bouwmeester, et al, Nature, 390, 575-579 (1997).

[3] A. Furusawa, et al. Science 282, 706-709 (1998).

[4] Pan, et al. Nature 421, 721-725 (2003).

[5] J. I. Cirac and A. S. Parkins, Phys. Rev. A 50, R4441 (1994).

[6] S. Bose, P. L. Knight, M. B. Plenio, and V. Vedral, Phys. Rev. Lett. 83, 5158 (1999).

[7] L. Davidovich, N. Zagury, M. Brune, J. M. Raimond, and S. Haroche, Phys. Rev. A 50, R895 (1994).

[8] S-B. Zheng, G-C. Guo, Phys. Rev. A 63, 044302(2001).

[9] W-L. Li, Ch-F. Li, G-C. Guo, Phys. Rev. A 61, 034301(2000).
[10] Z-L Cao and M. Yang, Physica A, 337/1-2, (2004)132140.

[11] Z-L Cao, M. Yang, Phys. Lett. A, 308(2003) 349-354.

[12] S. B. Zheng and G. C. Guo, Phys. Rev. Lett. 85, 2392 (2000).

[13] M. Riebe et al., Nature (London) 429, 734 (2004).

[14] M. D. Barrett et al., Nature (London) 429, 737 (2004).

[15] S. Osnaghi et al., Phys. Rev. Lett. 87, 037902 (2001).

[16] S. B. Zheng, Phys. Rev. A 69, 064302 (2004).

[17] Liu Ye and Guang-Can Guo, Phys Rev A 70, 054303 (2004)

[18] S.-B. Zheng, Phys. Rev. A 68, 035801 (2003). 